\title{
Design and Technology Challenges for Body Proximate Display Ecosystems
}

Jens Grubert

University of Passau

Passau, Germany

jg@jensgrubert.de

Matthias Kranz

University of Passau

Passau, Germany

matthias.kranz@uni-passau.de

Aaron Quigley

$\mathrm{SACHI}$

School of Computer Science

University of St Andrews, UK

aquigley@acm.org

\begin{abstract}
Body proximate display environments can be formed by combining multiple hand-held, head-mounted, wrist-worn or other displays. Wearable displays such as smartwatches and smartglasses have the potential to extend the interaction capabilities of mobile users beyond a single display. However, the display ecosystem formed by multiple personal displays on and around users' bodies is not well understood, yet. Within this paper, we investigate the design and technology challenges that could inhibit the creation and the use of interactive display ecosystems.
\end{abstract}

\section{ACM Classification Keywords}

H.5.m [Information interfaces and presentation]: Graphical user interfaces

\section{Introduction}

The proliferation of affordable consumer-oriented mobile and wearable displays, such as health bands (eg. fitbit or Microsoft Band), smartwatches, phablets, tablets, smartphones and smartglasses, potentially allow users to ac\footnotetext{
cess digital information in more situations than ever before. Some of these devices expect to operate in isolation, affording the user an "island of interaction", all too often locking their data into a silo. However, we are now seeing more of these devices being designed to work in device ecosystems, i.e. in conjunction with additional personal displays 


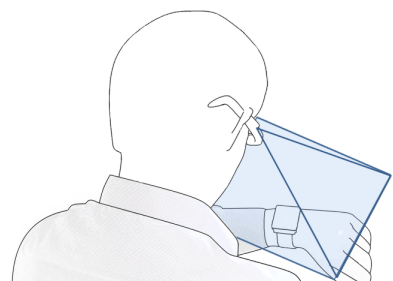

Figure 1: MultiFi [4], head-mounted display and smartwatch.

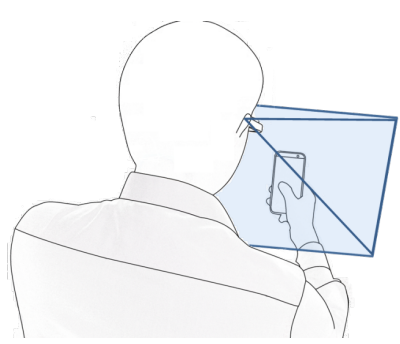

Figure 2: MultiFi [4], head-mounted display and smartphone.

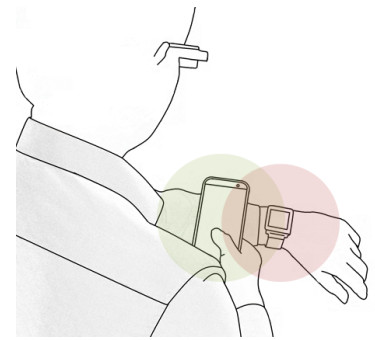

Figure 3: MultiFi [4], smartphone and smartwatch. with complementary device characteristics. Take, for example, smartwatches notifying their users about an incoming e-mail on their wrist but deferring the reading and writing of mails to an associated smartphone. Other devices can be appropriated to work in conjunction with additional displays. For example, a smartphone and a tablet can be used to distribute tasks across those devices (e.g., displaying a contact list on the mobile phone and an associated text document on the tablet). Smartglasses have the potential to show always-on information but, to date, are cumbersome to operate and can be subject to social challenges [6]. It has been shown that combinations of touch screens with smartglasses have the potential to lead to a more efficient interaction compared to smartlgass only interaction [?]. Many more permutations involving personal to public or appropriated to intimate displays are possible [11].

However, to date, what is not well understood is which factors inhibit or support the interaction across multiple displays on and around the body i.e. "body proximate" displays. Within this paper we review four key design and technological challenges inherent in body proximate display ecosystems, i.e. combinations of wearable displays (e.g., smartwatches and smartglasses) and handheld devices (e.g., tablets and smartphones).

\section{Design Challenges}

The fundamental design challenges in body-proximate display ecosystem reach beyond that of multi-display ecosystems [11]. Underlying factors are, amongst others, the reachability of displays (body-proximate displays are within arm's reach), perceptual characteristics (e.g., the costs of focus switch, c.f. [8]) or mobility aspects (usable while on-the-go). For the purpose of this paper we focus on the missing design factors and user interfaces widgets.
1. Missing Design Factors Several design factors for wearable multi-device interactions have been identified in previous work: groups explored how two touch screens could be used together by enabling or disabling their input and output channels, including combinations of smartphones with (large) interactive surfaces [10], smartwatches with interactive surfaces [5], or smartglasses with smartwatches [10], resulting in four different device combinations. Schmidt et al. [9] as well as Houben et al. [5] further refined how the interaction on a larger interactive surface could be parameterized by characteristics of the smartphone (respectively smartwatch), e.g., ID, pose, data context and (prior) selection on on the phone or smartwatch. Chen et al. extended frameworks for foreground-background interaction with individual devices [3] to a smartwatch - smartphone combination and explored interaction techniques when both the phone and the smartwatch were jointly used as foreground devices [1] (see Figure 4). Grubert et al. discussed further design dimension relevant for joint interaction between multiple wearable displays on and around the body, including smartglasses, smartphones and smartwatches (see Figures 1, 2 and 3): spatial reference frame, direct vs. indirect input, fidelity (i.e., the quality of output and input characteristics, such as resolution, color contrast, fixed vs. variable focus distance), continuity of fidelity (or fidelity gaps), continuity of the spatial reference frame and social acceptability of interactions [4]. In their work, Grubert et al. derived three alignment modes in which combinations of the wearable displays could be operated: body-aligned (i.e. displays are spatially registered and operate in a virtual information space that is fixed around the user's body), device-aligned (i.e. the information space is virtually registered to a touch display and virtually extended through the smartglass see Figure 5) and side-by-side (i.e. device combinations that do not require precise spatial alignment between devices see Figure 3). 
However, these works have only sampled the respective design spaces and combinations of design factors. Specifically, it remains unclear if the described design factors are sufficient for guiding future design space explorations, if and how they are interdependent, to which extend they are relevant for non-touch screen devices and how they scale to more than two jointly used displays. For example, fidelity gaps might be more relevant for touch-screen - smartglass interaction as the difference in output resolution and contrast is considerable larger compared to interaction with two touch screens only [4].

Further challenges for the interaction design of multiple wearable displays concern how to explicitly or implicitly transition between individual interaction modes, e.g., from side-by-side to device-aligned, from touch to mid-air interaction or when to switch the devices input and and output channels.

2. User Interface Widgets represent elements of interaction, yet it is unclear how these basic UI building blocks commonly found on mobile phones and smartwatches, lend themselves for transitioning between or spanning across multiple displays (including non-touch displays such as smartglasses). Also, it remains to be explored how to adopt a UI widget when it transitions from one display to another. Are design recommendation (e.g. from responsive web design) still valid when users relocate widgets frequently between displays?

\section{Technological Challenges}

There are a number of technological challenges to realise body proximate display ecosystems from optics, colour and contrast in hardware to synchronisation, middleware, data management, multi-model support in software. For the pur- poses of this paper we focus on mobile sensing and crossdevice developer toolkits.

3. Mobile Sensing Wearable displays such as smartphones and smartwatches typically allow for touch input on their interactive surface. Commercially available smartglasses often use indirect input via a touch pad. Sensing around individual devices has also been explored allowing above surface input on phones and smartwatches [5] or mid-air input in front of smartglasses (eg. Microsoft Hololens). Gestures using the devices themselves can also be realized, e.g., through inertial sensors or linear accelerometers. However, tracking the full six degrees of freedom poses of all multiple wearable devices, hence enabling a precise mutual spatial understanding of the display positions in space, has been so far restricted to lab-based prototypes. Approaches like MultiFi [4] typically rely on stationary optical outside-in tracking systems. We see the opportunity to combine device-integrated or body-mounted sensors into hybrid pose tracking systems in order to derive a full spatial understanding of all on-and around the body devices. However, to date it has not been explored how precise and reliable those mobile sensing solutions can work. Furthermore, it has still to be explored which granularity of spatial sensing (precise to none) is actually sufficient for various cross-device interaction tasks.

4. Cross-device Toolkits There is a growing number of development toolkits targeting cross-device applications involving mobile devices (e.g., [12, 7, 2, 5]). For example, they allow to distribute web-based user interfaces across displays with varying characteristics (such as size, distance, resolution) [12], allow for on-device authoring [7] or the integration of hardware sensor modules [5].

However, so far, those toolkits have mainly concentrated on 


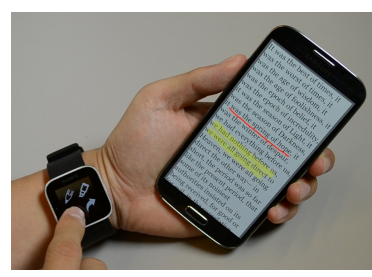

Figure 4: Duet [1], demonstrating coupled interactions on a smartphone and smartwatch.

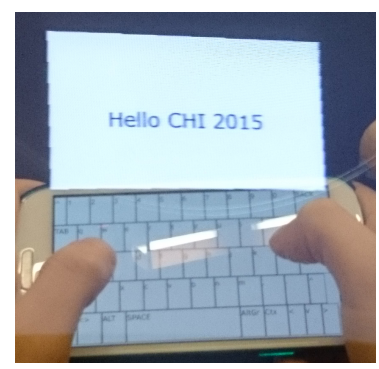

Figure 5: The screenspace of a smartphone is virtually extended with the help of a smartglass in MultiFi [4]. touch screen devices. More specifically, projection based systems, such as optical see-through head mounted displays (such as Google Glass, or Microsoft Hololens) have not been considered in the creation of those toolkits. Furthermore, authoring body-referenced information spaces, which float virtually around the users' body instead of coinciding with a physical screen [4], has not been in the focus of existing cross-device toolkits. Similarly, the specification of spatial gestures for triggering actions (e.g., through programming by example) has not been studied in this context. Finally, performance issues for web-based frameworks are still a hurdle to allow for fluid interaction across computationally restricted werable displays.

\section{Conclusion}

We see a limited appreciation of the relevant design dimensions to guide the creation of body proximate display applications. It remains unclear how to transition between individual interaction modes suggested in the literature. It is not well understood how user interface widgets should adopt their appearance and operation across displays with varying input and output characteristics. We have further identified the challenges presented in mobile sensing solutions for determining the spatial relationships between devices, and the missing consideration of non-touch screen displays in current cross-device developer toolkits. Through discussing these challenges we hope to contribute to shaping the research agenda of interaction with body proximate displays.

\section{REFERENCES}

1. X. Chen et al. 2014. Duet: exploring joint interactions on a smart phone and a smart watch. In Proc. CHI '14. 159-168.

2. P. Chi and Y. Li. Weave: Scripting Cross-Device Wearable Interaction. In Proc. CHI '15. ACM, New York, NY, USA, 3923-3932.
3. Hinckley et al. 2015. Foreground and background interaction with sensor-enhanced mobile devices. ACM TOCHI 12, 1 (2015), 31-52.

4. J. Grubert, M. Heinisch, and D. Quigley, A.and Schmalstieg. MultiFi: Multi Fidelity Interaction with Displays On and Around the Body. In Proc. $\mathrm{CHI}$ '15 (CHI '15). 3933-3942.

5. S. Houben and N. Marquardt. WatchConnect: A Toolkit for Prototyping Smartwatch-Centric Cross-Device Applications. In Proc. CHI '15. 1247-1256.

6. Marion Koelle, Matthias Kranz, and Andreas Moeller. 2015. Don't look at me that way! - Understanding User Attitudes Towards Data Glasses Usage. In Proc. MobileHCl' 15.

7. M. Nebeling, T. Mintsi, M. Husmann, and M. Norrie. Interactive Development of Cross-device User Interfaces. In Proc. CHI '14 (CHI '14). 2793-2802.

8. A. Quigley and J. Grubert. Perceptual and Social Challenges in Body Proximate Display Ecosystems. In Proc. MobileHCl '15 Adjunct. ACM.

9. D. Schmidt, J. Seifert, E. Rukzio, and H. Gellersen. A Cross-device Interaction Style for Mobiles and Surfaces. In Proc. DIS '12. 318-327.

10. M. Serrano et al. Smartwatches + Head-Worn Displays: the 'New' Smartphone. In CHI 2015 Adjunct.

11. L. Terrenghi, A. Quigley, and A. Dix. 2009. A Taxonomy for and Analysis of Multi-person-display Ecosystems. Personal Ubiquitous Comput. 13, 8 (Nov. 2009).

12. Jishuo Yang and Daniel Wigdor. Panelrama: enabling easy specification of cross-device web applications. In Proc. CHI '14. 2783-2792. 